\title{
Movimiento anti-vacunas
}

\section{Movement Anti-vaccines}

\author{
Diana V. Sanchez-Martinez ${ }^{a}$, Karla D. Figueroa-Almaraz ${ }^{b}$, Hazel M. Zamorano-Aguilar $^{c}$
}

\begin{abstract}
:
The "anti-vaccines" movement can be defined as a group of people who for different reasons (health, religious, scientific, political, philosophical) believe that vaccines and the act of getting vaccinated pose a greater risk to their health than the possible "benefit" they can contribute, spreading it through social networks, which nowadays are an easily accessible tool to share information of all kinds, including one that does not have an empirical-scientific foundation. The anti-vaccines movement is not homogeneous, since this expression is usually used to refer to different groups that are located in different points of what we might call a positioning before vaccination, that is, the postulates in the radical opposition to all vaccines, for the refusal, in a temporary or permanent sense of the parents towards their children, to administer one or several of the vaccines, for the damages derived from the vaccination " (Pareja, 2016)
\end{abstract}

\section{Keywords:}

Movement anti-vaccines, vaccination, social networks, positioning.

\section{Resumen:}

El movimiento "antivacunas" se puede definir como un colectivo de personas que por diferentes motivos (sanitarios, religiosos, científicos, políticos, filosóficos) creen que las vacunas y el acto de vacunarse supone un mayor riesgo para su salud que el posible "beneficio" que puedan aportar, difundiéndolo por redes sociales, las cuales hoy en día son una herramienta de fácil acceso para compartir información de todo tipo incluyendo aquella que no tiene una fundamentación empírica-científica. El movimiento antivacunas, no es homogéneo, ya que tal expresión se suele utilizar para hacer referencia a diferentes colectivos que se sitúan en distintos puntos de lo que podríamos denominar un posicionamiento ante la vacunación, es decir, los postulados en la oposición radical a todas las vacunas, por la negativa, en un sentido temporal o permanente de los padres hacia sus hijos, de administrar una 0 varias de las vacunas, por los daños derivados de la vacunación” (Pareja, 2016)

\section{Palabras Clave:}

Movimiento antivacunas, vacunación, redes sociales, posicionamiento.

\section{Introducción}

En el siguiente ensayo se expondrán diferentes puntos acerca de la importancia de la vacunación; para algunos quizá sea nueva y para otros ya tienen conocimientos previos. Donde se dará a conocer al lector el panorama que tienen el grupo de asociaciones "antivacunas", que se oponen a la vacunación por diferentes motivos.

El movimiento "antivacunas" se define como un colectivo de personas que por diferentes motivos (sanitarios, religiosos, científicos, políticos, filosóficos) creen que las vacunas y el acto de vacunarse suponen un mayor

\footnotetext{
a Autor de Correspondencia, Profesor por Asignatura de la Universidad Autónoma del Estado de Hidalgo, Escuela Superior de

Tlahuelilpan. E-mail: diana_sanchez8479@uaeh.edu,mx

b Alumno de la Universidad Autónoma del Estado de Hidalgo, Escuela Superior de Tlahuelilpan. E-mail:

karla.figueroaalmaraz@gmail.com
}

c Alumno de la Universidad Autónoma del Estado de Hidalgo, Escuela Superior de Tlahuelilpan. E-mail: hazelzamorano@gmail.com 
perjuicio para su salud que el posible beneficio que puedan aportar. Son grupos muy reivindicativos, que aportan información no contrastada y acientífica pero que ya han conseguido que las coberturas vacúnales en algunos países se hayan visto afectadas.

Es muy importante recalcar que se hablará de como este movimiento ha marcado una trayectoria internacional de casi dos siglos y con un fuerte arraigo en países del ámbito anglosajón, el cual ha causado preocupación en los profesionales sanitarios y sectores de salud por la aparición de cierto rechazo a vacunarse puede repercutir en la aparición de brotes epidémicos de enfermedades casi erradicadas, como la difteria, la tosferina o el sarampión.

\section{Desarrollo}

Desde el inicio de la vacunación, han existido grupos o movimientos, más o menos organizados, en contra de esta práctica, se da el caso de que estas circunstancias se han difundido con la irrupción y el éxito por las diferentes plataformas de internet y las nuevas posibilidades que ofrecen para la difusión de información a favor y en contra de la vacunación. Pero no hay que olvidar que todo esto está ocurriendo en un contexto social marcado por la pérdida de confianza en las instituciones públicas y el cuestionamiento del sistema socioeconómico... "como consecuencia, han surgido nuevas opciones políticas y parece extenderse la práctica de nuevos estilos de vida alternativos que, día a día, van consiguiendo más panoramas. Pero las más controversial es, el rechazo a las vacunas se puede definir como la negativa temporal o permanente de los padres a administrar una, varias o todas las vacunas a sus hijos" (Pareja, 2016)

Este tema como todo lleva un contexto histórico, pues la oposición a las vacunas es tan antigua como las mismas vacunas; un claro ejemplo es desde que en 1796 Edward Jenner, un médico rural inglés, empezó a utilizar un nuevo sistema para prevenir la viruela, por consecuente comenzaron a aparecer las primeras críticas y años más tarde los primeros movimientos sociales contrarios a la vacunación, gracias a esto se promulgan diferentes leyes que obligaban a vacunarse de la viruela en las que se llegaba a sancionar a los padres que no vacunasen a sus hijos con multas o incluso cárcel. Ante estas leyes se constituye en 1853 la Liga Antivacunación en Londres y más tarde en 1867 la Liga contra la vacunación obligatoria (Luna, 2015).

Otro claro ejemplo es en la década de los 70 donde surge en diversos países del mundo con una gran controversia con la vacuna frente a la difteria, tétanos y tosferina debido a un informe en el que se afirmaba que 36 niños habían sufrido consecuencias neurológicas (vómitos, irritabilidad, convulsiones, espasmos) después de recibir la vacuna. Debido a esto en Reino Unido disminuyó la tasa de vacunados y hubo tres epidemias de difteria. (Vazquez, 2017).

Pero sin importar los diferentes eventos que han ocurrido desde el principio que el movimiento antivacunas, no es homogéneo, sino que tal expresión se suele utilizar para hacer referencia a diferentes colectivos que se sitúan en distintos puntos de lo que podríamos denominar un posicionamiento ante la vacunación o sea que sus postulados es la oposición radical a todas las vacunas, por sus actitudes de rechazo a alguna vacuna en concreto, a las vacunas nuevas, o algún aspecto de ellas. ... "en la actualidad, aunque no se pueda generalizar porque este movimiento está más extendido en unos países que en otros, el rechazo en los países desarrollados se da mayoritariamente en familias con un nivel cultural superior a la media. Desde el punto de vista de los profesionales sanitarios, su preocupación es especialmente por tres argumentos muy utilizados por estos movimientos" (Quirós, 2014)

En primer lugar, que se niegue a que baje la incidencia de enfermedades infecciosas se debe a las vacunas y que, en cambio, se atribuya a la mejora de las condiciones socioculturales y sanitarias. Otro motivo, es que estos grupos suelen utilizar como prueba: ejemplos y estadísticas de personas vacunadas que, a pesar de ello, contrajeron la enfermedad. Pero el argumento más repetido, según el citado el autor, es ir sumando alteraciones graves y enfermedades en niños vacunados coincidiendo su diagnóstico en el tiempo de la administración de la vacuna.

Los primeros síntomas de arraigo del movimiento antivacunas en España ya son motivo de preocupación para los profesionales de la salud, y es precisamente desde este ámbito los expertos vienen proponiendo una serie de recomendaciones...."las nuevas estrategias que se plantean van dirigidas a los padres y pacientes, pero también a todos los agentes sociales implicados en la vacunación: a los profesionales sanitarios, al binomio sistemas de salud-fabricantes y a los medios de comunicación" (Marco \& Zamanillo Rojo, 2003)

\section{Conclusiones}

Para concluir se debe mencionar que es necesario revisar algunas cuestiones específicas, como la información que brinda el personal de salud a la sociedad acerca de las vacunas y la seguridad de estas, pero más importante es realizar mejoras en el ámbito sanitario, por ejemplo, en los registros de seguimiento de las vacunas, aplicación y 
monitoreo de las campañas, pues exigen un compromiso profesional para que la práctica informativa genere confianza a la población. La democracia como la ciencia seguirá evolucionando y se debe asegurar que dicha evolución suponga una mejora que redunde positivamente en la convivencia y el bienestar de esta generación y de las futuras.

...”la realización de más estudios independientes en seguridad vacunal, a la mejora del sistema de monitorización de los efectos adversos, a la accesibilidad a dichos datos y a la creación de un fondo compensatorio por daños derivados de la vacunación" (Tuells, 2016)

\section{Referencias}

Iván Renato Zúñiga Carrasco, J. C. (2018).Grupos antivacunas: el regreso global de las enfermedades prevenibles. Revista Latinoamericana de Infectología Pediátrica. Volumen 31, Número 1 Enero-Marzo pp. 17-21.

Luna, S. M. (2015). Los Movimientos Antivacunas y su presencia en el Internet. Revista de enfermeria Vol 9, No 3.

Pareja, E. H. (2016). El movimiento antivacunas Argumentos, Causas y Consecuencias. Madrid: Los Libros de la Catarata.

Rodrigo, M. A. (2015). Antivacunas: un reto para el pediatra. Pediatría Atención Primaria. Vol.17 no.66 abr./jun..

Vazques, S. H. (2017). Controversia de la vacuna Td. Revista Chilena de Derecho, 563-573. 